\title{
Optimized Waveform for Energy Efficient Ranging
}

\author{
Emanuel Staudinger, Michael Walter, and Armin Dammann \\ German Aerospace Center (DLR) \\ Institute of Communications and Navigation \\ Oberpfaffenhofen, 82234 Wessling, Germany \\ Email: \{emanuel.staudinger\}@dlr.de
}

\begin{abstract}
Mobile communication terminals exploit existing reference signal structures for propagation delay based positioning. However, the used waveforms are not optimized for energy efficiency and improved ranging performance for positioning. Recently, a parametric waveform with adaptable power spectral density has been proposed in the context of $5 \mathrm{G}$, and has shown an improved ranging performance.

In this paper, we investigate the energy reduction of a ranging signal for a targeted ranging performance by adjusting the parametric waveform. We focus on the newly opened $28 \mathrm{GHz}$ frequency band offering $850 \mathrm{MHz}$ of contiguous bandwidth in the United States. Based on derived Ziv-Zakai lower bounds and a mmWave path loss model with shadow fading we determine the optimal waveform parameter. Our results show a transmit power reduction of $4.77 \mathrm{~dB}$ compared to existing reference signal structures. Furthermore, we show a link budget example in the context of ITS positioning.
\end{abstract}

\section{INTRODUCTION}

Nowadays, parameters of future communication systems such as $5 \mathrm{G}$ and for intelligent transportation systems (ITSs) are intensively discussed. At the moment, infrastructure based communication technologies like WiFi, ITS-G5, UWB tracking, Bluetooth, and 3GPP-LTE are used for positioning. For example in the European HIGHTS project the afore mentioned technologies are investigated particularly for ITS [1]. The next major step will be the $5 \mathrm{G}$ standard, and various research questions related to throughput, reliability, and latency are currently investigated [2]-[4]. Besides requirements related to communications, network based positioning should be supported, as global navigation satellite system (GNSS) localization performance is degraded in typical urban scenarios due to multipath, shadowing, and unfavorable geometries. How can positioning benefit from 5G? The expected benefit will be larger bandwidths at higher carrier frequencies for higher ranging accuracy, phased arrays at higher carrier frequencies for angle of arrival determination, and flexible multi-carrier waveforms. 5G will also enable device-to-device (D2D) connectivity, paving the way for cooperative positioning techniques.

Fig. 1 shows an exemplary ITS scenario used in this paper. Each mobile vehicle and vulnerable road user has a radio device attached: this device is commonly used to connect to a base station or a so called road side unit for communication purposes. As D2D becomes possible in $5 \mathrm{G}$, all entities on the road are able to communicate with their respective neighbors. Reliable location information of each entity on the road is of utmost importance for, e.g., future automated driving.

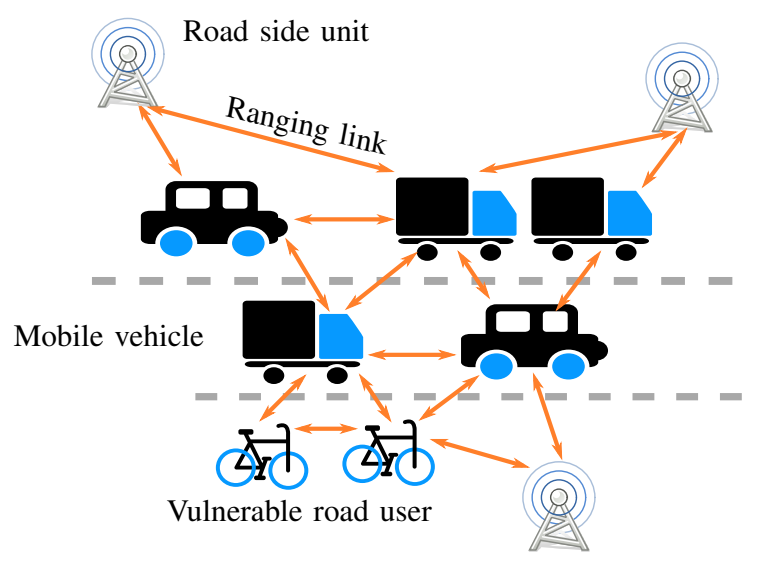

Fig. 1. Example scenario for cooperative ITS positioning involving multiple entities equipped with a radio device: cars, trucks, vulnerable road users such as cyclists, and infrastructure such as road side units. Orange arrows indicate a communication and ranging link between the different entities on the road and to the road side units. Cooperative localization benefits from improved ranging accuracy and dense networks, and requires local communication only. Hence, 5G technologies could enable cooperative localization for all entities on the road.

Consequently, we can introduce a propagation delay based ranging capability, and are able to cooperatively determine the position of each entity in this adhoc network. Ranging performance will depend on channel conditions between mobiles and the particular waveform used for the reference signal. $5 \mathrm{G}$ adopts multi-carrier signals with flexible parameters, and hence, parametric waveforms for ranging can be used.

In 2016 the Federal Communications Commission (FCC) reported a proposed rulemaking to open two contiguous bandwidth-blocks of $425 \mathrm{MHz}$ in the $28 \mathrm{GHz}$ frequency band, which shall be licensed on a county-wise level [5]. This large bandwidth of $850 \mathrm{MHz}$ will result in a significantly improved ranging performance. The $28 \mathrm{GHz}$ frequency band permits short communication ranges only, assuming typical mobile transmit powers and omnidirectional antennas. However, cooperative positioning works with local communication and ranging only. As a result, the spatial re-use of spectral resources at this frequency band will be higher compared to lower frequency bands.

We investigate in this paper in a first step the achievable ranging performance in the afore mentioned frequency band, taking a path loss model with shadow fading into account. In a second step we define a cost function based on the Ziv-Zakai 
lower bound (ZZLB) to jointly determine the lowest required signal-to-noise ratio (SNR) and optimal waveform parameter to reach a specific ranging root mean square error (RMSE). By comparing the newly required SNR with the necessary SNR of a state of the art reference signal for ranging, we can show a gain resulting in a more energy efficient mobile system.

This paper is structured as follows: the propagation model is described in Sec. II, and we recall one parametric waveform with the used transmission model and derived lower bound in Sec. III. Furthermore, we determine the ranging performance with shadow fading included. In Sec. IV we define our new cost function and show results for joint signal power and parameter optimization to reach a targeted ranging RMSE. We give a practical link budget example for ITS positioning in Sec. $\mathrm{V}$ and show the benefit of our proposed approach to significantly reduce the energy consumption of future localization systems. Sec. VI shortly addresses possible future work and concludes the paper.

\section{Propagation Environment}

Various mmWave channel models based on channel sounding measurements have been published recently, covering frequency bands between $28 \mathrm{GHz}$ and $73 \mathrm{GHz}$ [6]-[8]. We are particularly interested in the line-of-sight (LoS) path loss at $28 \mathrm{GHz}$ between a base station (BS) and a mobile vehicle (MV), as well as between MVs. Recent path loss models at $28 \mathrm{GHz}$ primarily focus on transmitter-receiver setups with directed antennas: in most cases horn antennas with high antenna gain. However, as we focus on single antenna systems without directivity we require an omnidirectional path loss model. The omnidirectional LoS path loss with shadow fading at $28 \mathrm{GHz}$ is given by the deterministic function according to [9] as

$$
\mathrm{PL}[\mathrm{dB}](d)=61.4+21 \log _{10}(d)+\mathrm{SF},
$$

with $d$ as LoS distance between transmitter and receiver. This model also includes shadow fading

$$
\mathrm{SF}[\mathrm{dB}] \sim \mathcal{N}\left(0, \sigma_{\mathrm{SF}}^{2}\right),
$$

which is a random variable and is drawn in $\mathrm{dB}$ from a normal distribution with zero mean and standard deviation $\sigma_{\mathrm{SF}}$. The shadow fading standard deviation in this paper is $\sigma_{\mathrm{SF}}=3.6 \mathrm{~dB}$ [9]. At this point we need to remark that the LoS path loss model (1) from [9] is derived from measurements at only five spatial points, see [10] for details.

\section{WAVEForm Definition, Transmission Model, AND PERFORMANCE BOUNDS}

In general there is a trade-off between delay estimation resolution and detection ambiguities for propagation delay based ranging. For any given SNR a dedicated waveform power spectral density (PSD) exists, which minimizes the mean-square error (MSE) for range estimation. The authors in [11] proposed several single-parametrized ranging waveforms with different PSDs, which can also be viewed as windowing functions. For completeness we recall the so called DiracRectangular waveform, transmission model, and the derived

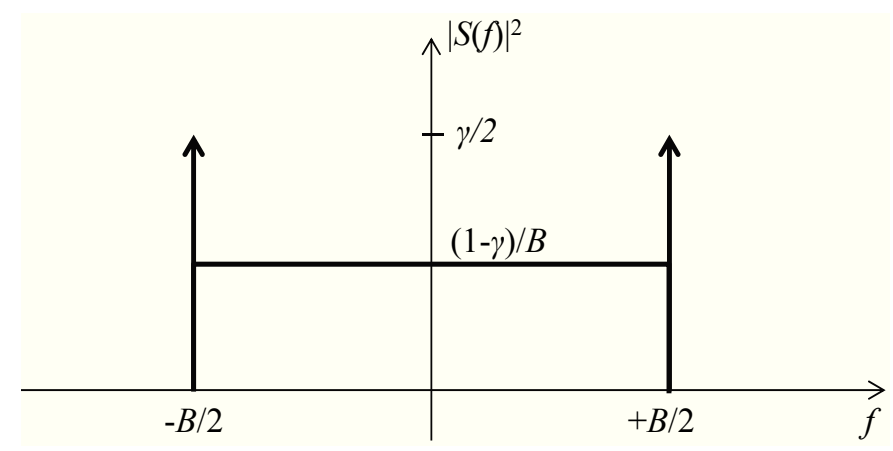

(a) Power spectral density $|S(f)|^{2}$.

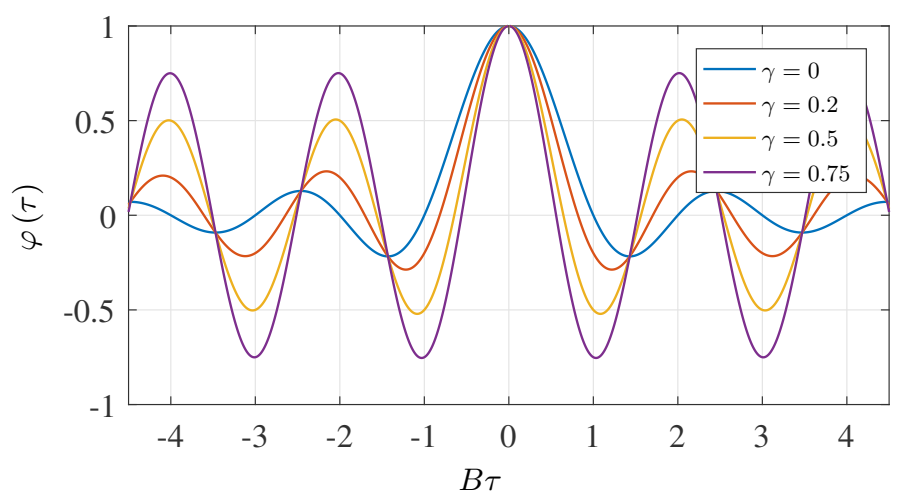

(b) Autocorrelation function $\varphi(\tau)$.

Fig. 2. Dirac-rectangular waveform [11].

lower bound from [11] in this section. Additionally, we extend the contribution from [11] with the path loss model and shadowing factor from Sec. II.

\section{A. Dirac-Rectangular Waveform}

In this paper we consider a single-parametrized, bandlimited waveform of bandwidth $B$. The waveform is a weighted superposition of two Dirac delta functions at the spectrum's edge and a rectangular PSD, see Fig. 2(a). We refer to this waveform as Dirac-rectangular waveform with its PSD denoted as

$$
|S(f)|^{2}= \begin{cases}\frac{1-\gamma}{B}+\frac{\gamma}{2}\left[\delta\left(f+\frac{B}{2}\right)+\delta\left(f-\frac{B}{2}\right)\right], & |f| \leq \frac{B}{2} \\ 0, & |f|>\frac{B}{2}\end{cases}
$$

The Dirac delta function is represented by $\delta$, and $\gamma \in \mathbb{R}, 0 \leq$ $\gamma \leq 1$ denotes the so-called waveform shaping parameter. The parameter $\gamma$ determines the trade-off between delay estimation resolution and detection ambiguities. For $\gamma=0$ we obtain a state of the art rectangular PSD, and $\gamma=1$ results in a signal power concentration at the edges of the spectrum. The corresponding autocorrelation function as the inverse Fourier transform of the PSD calculates to [11]

$$
\varphi(\tau)=(1-\gamma) \frac{\sin (\pi B \tau)}{\pi B \tau}+\gamma \cos (\pi B \tau)
$$

Fig. 2(b) shows autocorrelation functions for specific values of $\gamma$. With an increasing $\gamma$ the signal power is more concentrated 
at the edges of the spectrum, which leads to a tighter mainlobe at the cost of higher side-lobes in the autocorrelation function. Hence, we can choose between ranging precision and mis-detection for further optimization.

\section{B. Transmission Model}

We consider a transmission model comprising a multicarrier transmitter and a multi-carrier receiver based on orthogonal frequency division multiplex (OFDM). Transmitter and receiver are perfectly synchronized to enable time-of-arrival (ToA) or time-of-flight (ToF) based ranging. The received signal is

$$
r[n]=\alpha s\left(n-\tau / T_{s}\right)+z[n],
$$

with $\alpha$ as instantaneous flat fading coefficient and $\tau$ as propagation delay according to $\tau=d / c_{0}$ with $d$ as the LoS distance in meters and $c_{0}$ as the speed of light. $z[n]$ represents white Gaussian distributed noise with zero mean and variance $N_{0}$, and $T_{s}$ denotes the sampling time interval. The instantaneous fading coefficient with (1) is defined as

$$
\alpha=10^{-\frac{\mathrm{PL}}{20}},
$$

and the resulting instantaneous SNR after the matched filter in the receiver yields

$$
\mathrm{SNR}=\frac{E_{s}}{N_{0}}=\frac{P_{\mathrm{Tx}} \alpha}{N_{0}} .
$$

$P_{\text {Tx }}$ denotes the transmit power and we assume unity antenna gain for transmitter and receiver respectively.

\section{Range Estimation Performance Bounds}

A common method to quantify precision of an unbiased estimator is the Cramér-Rao lower bound (CRLB). For propagation delay based range estimation the CRLB is stated as [12]

$$
\sigma_{\text {CRLB }}^{2}\left[\mathrm{~m}^{2}\right]=\frac{c_{0}^{2}}{8 \pi^{2} \beta^{2} \frac{E_{s}}{N_{0}}},
$$

with $\sigma_{\text {CRLB }}^{2}$ as the theoretical ranging variance, given a particular SNR of $E_{s} / N_{0}$ after the matched filter, and the so-called effective or equivalent signal bandwidth $\beta^{2}$. For the Diracrectangular waveform the equivalent signal bandwidth states

$$
\beta^{2}=\frac{\int f^{2}|S(f)|^{2} \mathrm{~d} f}{\int|S(f)|^{2} \mathrm{~d} f}=\frac{B^{2}}{12}(1+2 \gamma),
$$

with $B$ as signal bandwidth from (3) and the waveform shaping parameter $\gamma$. A larger $\gamma$ results in a larger $\beta^{2}$ yielding a lower CRLB, but at the cost of higher autocorrelation function side-lobes, see Fig. 2(b). At very low SNRs, an estimator might erroneously select a side-lobe with certain probability and interpret it as main-lobe. Hence, the ranging variance increases significantly for low SNRs which is also known as waterfall region, as the CRLB is tight for reasonably high SNRs only. In order to account for mis-detections at low SNR regions we consider the ZZLB and follow results in [13]

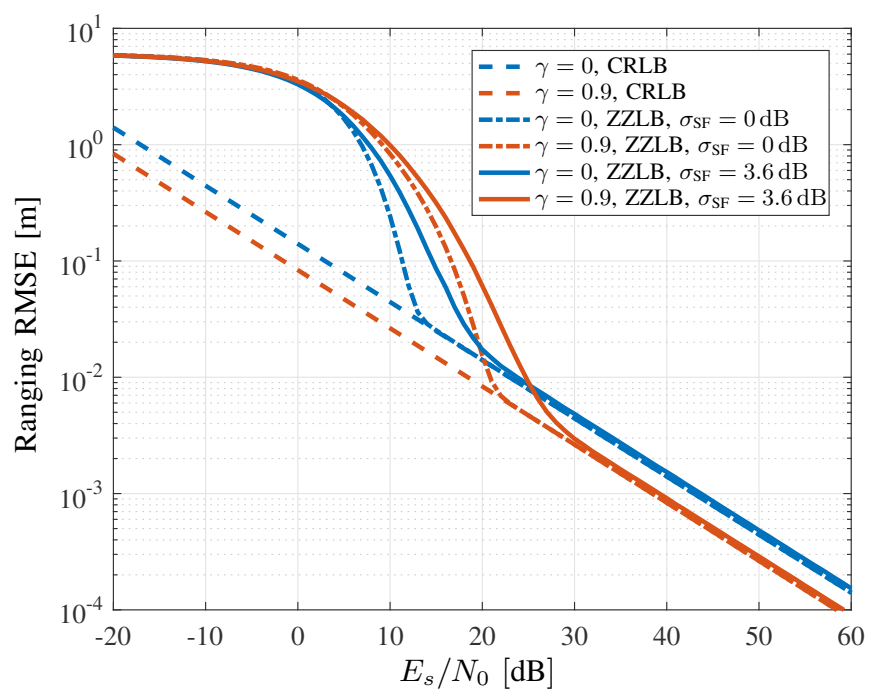

Fig. 3. Square root of the CRLB and ZZLB for range estimation using Diracrectangular waveforms with two particular shaping parameters $\gamma$. Additionally, the ZZLB including shadow fading $\left(\sigma_{\mathrm{SF}}=3.6 \mathrm{~dB}\right)$ is shown and we clearly see a difference in the SNR threshold region compared to the case without shadow fading $\left(\sigma_{\mathrm{SF}}=0 \mathrm{~dB}\right) . E_{s} / N_{0}$ represents the SNR after the matched filter.

for this type of lower bound. Accordingly, the ZZLB for range estimation calculates to

$\sigma_{\text {ZZLB }}^{2}\left[\mathrm{~m}^{2}\right]=c_{0}^{2} \int_{0}^{T_{\text {obs }}} \tau\left(1-\frac{\tau}{T_{\text {obs }}}\right) \phi\left(\sqrt{\frac{E_{s}}{N_{0}}(1-\varphi(\tau))}\right) \mathrm{d} \tau$,

where $\varphi(\tau)$ denotes the autocorrelation function from (4), and $\phi$ the Gaussian Q-function

$$
\phi(x)=\frac{1}{\sqrt{2 \pi}} \int_{x}^{\infty} \mathrm{e}^{-\frac{t^{2}}{2}} \mathrm{~d} t .
$$

Parameter $T_{\mathrm{obs}}$ describes the length of an observation interval within the parameter $\tau$ is equally distributed. In practice, the length of an observation interval for OFDM modulated signals would be either one half of the symbol length or the length of the cyclic prefix. The latter case assumes a coarse pre-synchronization which is inherently required for a communication receiver. Furthermore, the ZZLB in (10) represents the instantaneous variance only, as the signal power $E_{s}$ from (7) includes $\alpha$ as random variable. Consequently, we need to calculate the expectation value of (10).

\section{Resulting Ranging RMSE with shadow fading}

We now have a closer look at the resulting performance bounds for one specific ITS setup: we assume a short OFDM symbol comprising 128 subcarriers with a total bandwidth of $850 \mathrm{MHz}$, and an observation interval $T_{\text {obs }}$ of 60 samples. Fig. 3 shows the square root of the CRLB and the ZZLB with and without shadow fading respectively. For simplicity we only show results for two waveform shaping parameters: $\gamma=0$ for the purely rectangular waveform, and $\gamma=0.9$. The threshold effect of the ZZLB between an SNR of $10 \mathrm{~dB}$ and 
$30 \mathrm{~dB}$ is clearly visible. For $\gamma=0.9$ we observe a lower CRLB at higher SNRs due to the larger equivalent signal bandwidth but mis-detections due to autocorrelation function ambiguities occur at higher SNR values as well. The dashdotted lines show the resulting ZZLB with shadow fading taken into account. Compared to the ZZLB result without the shadow fading from [11] we observe a larger range of SNR values for the waterfall region, i.e., for $\gamma=0.9$ the waterfall region starts at an SNR of about $30 \mathrm{~dB}$ compared to an SNR of about $21 \mathrm{~dB}$ for $\sigma_{\mathrm{SF}}=0 \mathrm{~dB}$.

This first result also shows that we can reach ranging RMSEs below $1 \mathrm{~mm}$ due to the large available bandwidth. However, a larger SNR is required if we take shadow fading into account to remain tight to the CRLB compared to the ideal case without shadow fading. A small gap between the ZZLB with and without $\sigma_{\mathrm{SF}}$ is visible at high $E_{s} / N_{0}$. This gap results from Jensen's inequality, as we need to calculate the expectation value over the instantaneous ZZLB with shadow fading [14].

In most cases we are interested in obtaining the optimal waveform parameter to achieve the lowest possible ranging RMSE for a given SNR. In this work we assume a specific required ranging RMSE for, e.g., an ITS application and aim to find the lowest required SNR jointly with the optimal waveform parameter. Hence, the cost function for minimization must be reformulated compared to our previous work [11]. A lower required SNR consequently yields a lower required transmit power compared to state of the art, resulting in a more energy efficient mobile system.

\section{Optimal WAVEForm Parameter FOR Transmit POWER REDUCTION}

We introduce the term targeted ranging RMSE, which defines a particular ranging RMSE $\sigma_{\mathrm{T}}$ we seek to achieve, i.e., a $\sigma_{\mathrm{T}}$ of $1 \mathrm{~cm}$ might be sufficient for many applications. Assuming a fixed signal bandwidth, we have to determine the lowest required SNR and optimal waveform parameter $\gamma$ to reach $\sigma_{\mathrm{T}}$. Hence, we need to reformulate our cost function for a two-dimensional optimization. Once an optimal SNR and shaping parameter tuple has been found, we can give a realistic ITS example based on a link budget assessment.

The joint optimization function with respect to the SNR and the waveform shaping parameter $\gamma$ is defined as

$$
\mathrm{SNR}_{\gamma_{\mathrm{opt}}}=\underset{\mathrm{SN}, \tilde{\gamma}}{\arg \min }\left|\sigma_{\mathrm{T}}-\mathbb{E}\left\{\sqrt{\sigma_{\mathrm{ZZLB}}^{2}(\mathrm{SNR}, \tilde{\gamma})}\right\}\right|,
$$

resulting in $\mathrm{SNR}_{\gamma_{\text {opt }}}$ as the optimized SNR under the constraint of the optimal $\gamma$. The expectation value in (12) is required, as the SNR definition in (7) includes the fading coefficient $\alpha$ as random variable. Fig. 4 illustrates an example of the optimization function for a targeted ranging RMSE of $\sigma_{\mathrm{T}}=0.01 \mathrm{~m}$. At first we determine the required $\mathrm{SNR}_{\gamma=0}$ for the state of the art rectangular waveform and use (12) with a fixed $\tilde{\gamma}=0$. The solid blue line in Fig. 4 shows the resulting ranging RMSE for that determined $\mathrm{SNR}_{\gamma=0}$ if we additionally increase the shaping parameter. As expected, the ranging RMSE decreases

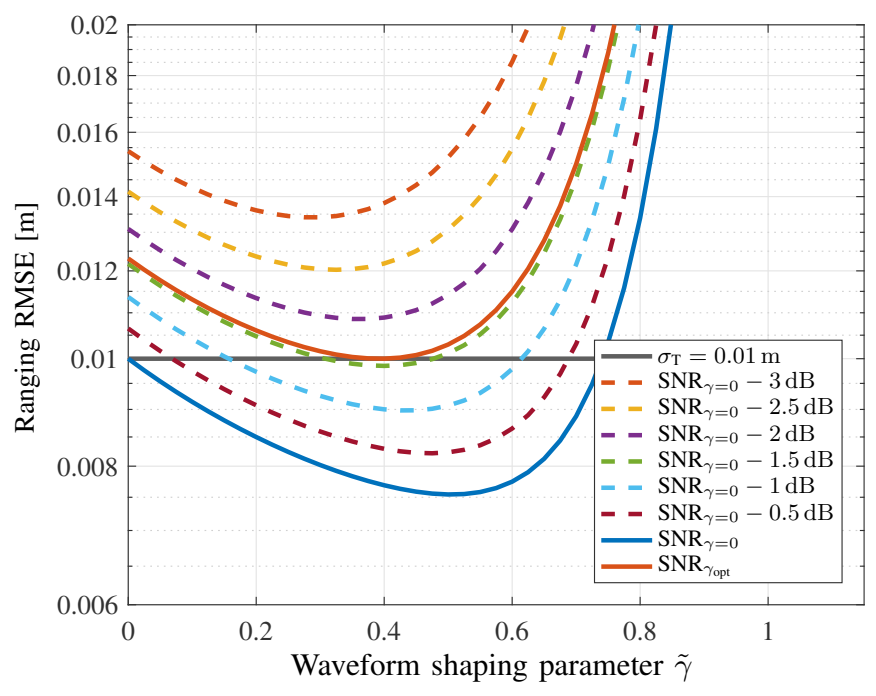

Fig. 4. Illustration of the ranging RMSE over the waveform parameter $\gamma$ for different SNRs. The target ranging RMSE $\sigma_{\mathrm{T}}$ is $0.01 \mathrm{~m}$ which can be reached with different SNR and $\gamma$ combinations. The solid red line shows the optimized case with the lowest required SNR reaching $\sigma_{\mathrm{T}}$ under the constraint of using the optimal waveform parameter $\gamma_{\mathrm{opt}}$.

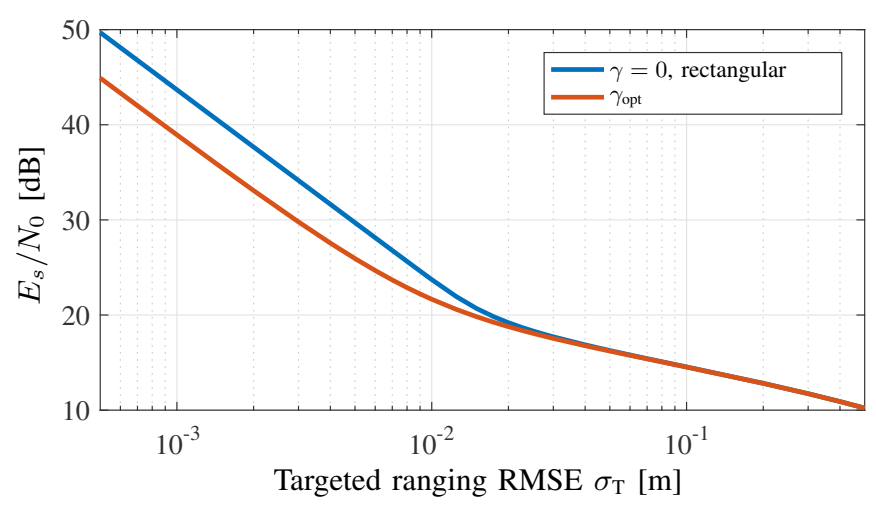

Fig. 5. Required SNR for a rectangular waveform $(\gamma=0)$ and optimized waveform $\left(\gamma_{\mathrm{opt}}>0\right)$ to achieve a specific targeted ranging RMSE $\sigma_{\mathrm{T}}$. A shadow fading factor of $\sigma_{\mathrm{SF}}=3.6 \mathrm{~dB}$ is included. $E_{s} / N_{0}$ represents the SNR after the matched filter.

with the shaping parameter $\tilde{\gamma}$ reaching a minimum at $\tilde{\gamma} \approx 0.55$. The dashed lines in Fig. 4 show the resulting RMSE curves if we consecutively reduce the initially determined $\mathrm{SNR}_{\gamma=0}$ in $0.5 \mathrm{~dB}$ steps. We can observe that the initially determined $\mathrm{SNR}_{\gamma=0}$ can be reduced significantly. Once the SNR is too small, we can not reach the targeted $\sigma_{\mathrm{T}}$ with any $\tilde{\gamma}$. The solid red line shows the final result from (12) in this example: compared to the state of the art rectangular waveform we can reduce the required SNR by about $1.7 \mathrm{~dB}$ by using a $\gamma_{\text {opt }}$ of 0.4 .

At this point we need to remark that the ranging RMSE curves in Fig. 4 are smooth which is only the case for larger $\sigma_{\mathrm{T}}$. For smaller targeted $\sigma_{\mathrm{T}}$ the optimal shaping parameter $\tilde{\gamma}$ converges to 1 resulting in a sharp transition of the ranging RMSE at parameter values close to 1 . 


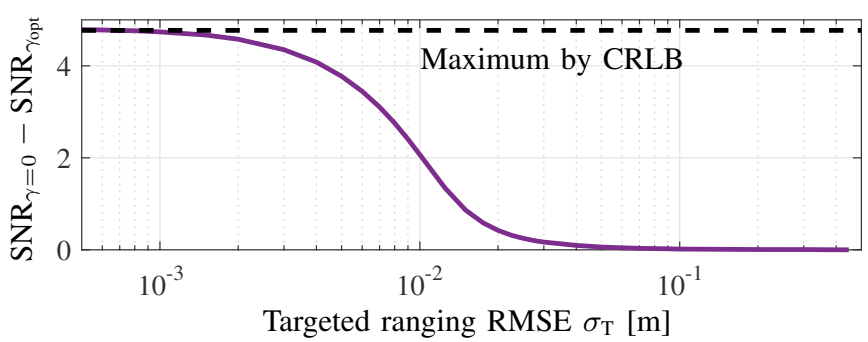

(a) Resulting SNR difference between the state of the art rectangular waveform and the optimal waveform for a specific targeted ranging RMSE. The dashed line shows the theoretically achievable maximum SNR difference, see (9).

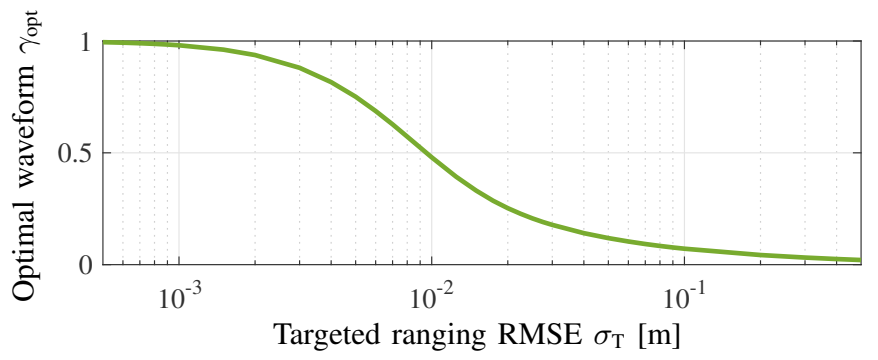

(b) Optimal waveform parameter $\gamma_{\text {opt }}$ for a specific targeted ranging RMSE.

Fig. 6. Results from the joint SNR and waveform parameter optimization. A gain in SNR is clearly visible.

Fig. 5 shows the required SNR for the state of the art rectangular waveform over a wide region of targeted ranging RMSEs $\sigma_{\mathrm{T}}$. A bend in required SNR above $\sigma_{\mathrm{T}}=0.01 \mathrm{~m}$ results from increasing incorrect detections of the main autocorrelation lobe, see also Fig. 3. The solid red line shows the required SNR under the constraint of the optimal shaping parameter. A significant difference in SNR is visible, particularly at low targeted ranging RMSEs.

Fig. 6(a) shows the resulting SNR difference, respectively the gain. For large $\sigma_{\mathrm{T}}$ the SNR gain vanishes, as the ranging RMSE lies in the waterfall region of the ZZLB. For small $\sigma_{\mathrm{T}}$ we get an SNR difference which converges to the maximum achievable gain of $4.77 \mathrm{~dB}$. Hence, by using an optimized waveform we can reduce the required transmit power by $4.77 \mathrm{~dB}$. This maximum can also be observed from the CRLB, see (9). The corresponding optimal waveform parameter $\gamma_{\mathrm{opt}}$ is shown in Fig. 6(b).

\section{LINK BUDGET EXAMPLE FOR ITS POSITIONING}

In the previous section we have shown the two-dimensional cost function to determine the required SNR and optimal waveform parameter $\gamma$ to achieve a particular targeted ranging RMSE $\sigma_{\mathrm{T}}$. Next we have a closer look on how to use the previously described results to obtain tangible numbers from a link budget assessment.

Parameters for the link budget calculation are as follows: we assume an OFDM signal with a bandwidth of $850 \mathrm{MHz}$ at a carrier frequency of $28 \mathrm{GHz}$. The radio channel between mobile vehicles exhibits a strong dynamic (time varying) behavior. Consequently, we use a short OFDM signal with

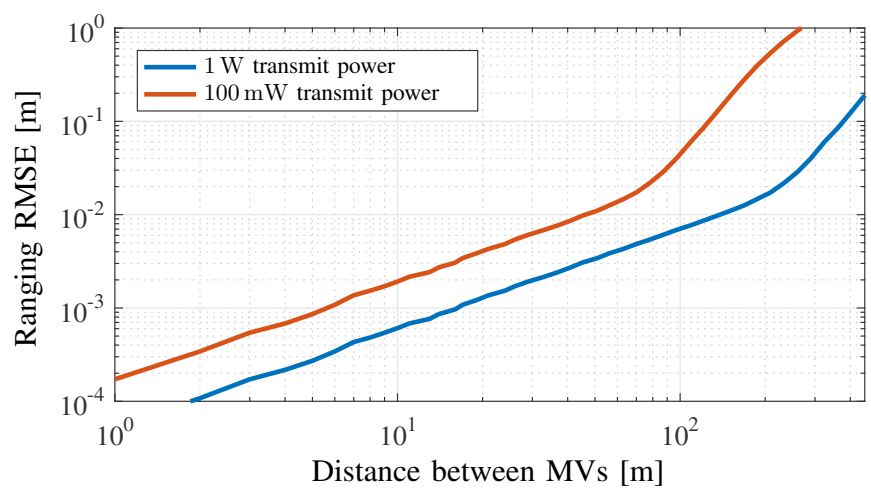

Fig. 7. Ranging RMSE over distance at a carrier frequency of $28 \mathrm{GHz}$, with a bandwidth of $850 \mathrm{MHz}$, and with two different transmit powers. The waveform shaping parameter $\gamma$ is set to 0 to show the baseline ranging performance with a rectangular waveform.

128 subcarriers only, and an observation interval $T_{\text {obs }}$ of 60 samples for the ZZLB. For the SNR as defined in (7) we include the correlation gain of the 128 samples long OFDM signal, thermal noise with a temperature of $300 \mathrm{~K}$, and an additional noise figure of $5 \mathrm{~dB}$. We take the path loss model with shadow fading from (1) into account, and obtain the lower bound for a state of the art rectangular waveform with $\gamma=0$ according to (10). Fig. 7 shows the resulting ranging RMSE for a transmit power of $1 \mathrm{~W}$ and $100 \mathrm{~mW}$ respectively. A ranging RMSE of less than $1 \mathrm{~cm}$ at a distance of $100 \mathrm{~m}$ is possible, despite the large path loss at a carrier frequency of $28 \mathrm{GHz}$ with assumed omni-directional antennas.

Fig. 7 shows the baseline ranging performance with a state of the art waveform. Let us assume we require a targeted ranging RMSE $\sigma_{\mathrm{T}}$ of $5 \mathrm{~mm}$ at a distance of $40 \mathrm{~m}$ for a particular ITS positioning application. Thus, we have a look at Fig. 5 and Fig. 6(b). For $\gamma=0$ we obtain a necessary SNR of $29.71 \mathrm{~dB}$. Based on our optimized waveform we only require an SNR of $25.93 \mathrm{~dB}$ with a Dirac-rectangular waveform and a shaping parameter $\gamma=0.75$. We take the previous link budget parameters into account and obtain the following transmit powers: Using a state of the art waveform we need a transmit power of $242.4 \mathrm{~mW}$ compared to $101.5 \mathrm{~mW}$ for the optimized waveform. The benefit of a lower transmit power is twofold: firstly, the obvious energy consumption as the transmitted signal requires less energy. Secondly, the radio frequency (RF) power amplifiers can be smaller. Particularly for multi-carrier signals one requires less efficient linear power amplifiers due to the high crest-factor. Using smaller RF power amplifiers, as less RF output power is needed, reduces the overall energy consumption of a mobile system even more. The parametric waveform considers the PSD only, leaving an additional degree of freedom to reduce the crest-factor of a multi-carrier signal by adapting the subcarrier phases.

\section{SUMMARY AND OUTLOOK}

We have shown the possibility of transmit power reduction for ITS positioning by jointly determining the optimal parameter and required SNR of a parametric waveform for 
ranging. Our numerical evaluations based on the ZZLB show a power reduction of up to $4.77 \mathrm{~dB}$. A link budget calculation for ranging at $28 \mathrm{GHz}$ with a bandwidth of $850 \mathrm{MHz}$ shows that ranging RMSEs down to centimeters are possible for distances up to $100 \mathrm{~m}$.

In this work we only had have a look at the transmit power reduction and assume a single OFDM symbol solely for ranging. Communication systems with short packets might not spare a single symbol for ranging only, e.g., multiplexing data carriers with the pilots (reference signal structure) might be desired. In [15] we introduced the concept of sparse subcarrier allocation for ranging with OFDM signals. Consequently, future work should address sparse subcarrier allocation evaluated with the ZZLB. This approach frees spectral resources for data carriers in the same OFDM symbol by reaching a specific targeted ranging RMSE at the same time.

\section{ACKNOWLEDGMENT}

This work has been performed in the framework of the German Aerospace Center (DLR) project Navigation 4.0, and has been partially supported by the EU project HIGHTS (High precision positioning for cooperative ITS applications) MG3.5a-2014-636537.

\section{REFERENCES}

[1] "HIGHTS Deliverable D5.1: Cooperative Localization Algorithms and Hybrid Data Fusion Schemes," Jun. 2016. [Online]. Available: http://hights.eu/?page_id=699

[2] G. Fettweis and S. Alamouti, "5g: Personal mobile internet beyond what cellular did to telephony," IEEE Commun. Mag., vol. 52, no. 2, pp. 140 - 145, Feb. 2014.

[3] J. G. Andrews, S. Buzzi, W. Choi, S. V. Hanly, A. Lozano, A. C. K Soong, and J. C. Zhang, "What will $5 \mathrm{~g}$ be?" IEEE J. Sel. Areas Commun., vol. 32, no. 6, pp. 1065 -1082, Jun. 2014.
[4] P. Banelli, S. Buzzi, G. Colavolpe, A. Modenini, F. Rusek, and A. Ugolini, "Modulation formats and waveforms for $5 \mathrm{~g}$ networks: Who will be the heir of ofdm?: An overview of alternative modulation schemes for improved spectral efficiency," IEEE Signal Process. Mag., vol. 31, no. 6, pp. $80-93$, Nov. 2014.

[5] F. C. Commission, "Report and order of further notice of proposed rulemaking, gn docket no. 14-177," Jul. 2016.

[6] T. S. Rappaport, G. R. MacCartney, M. K. Samimi, and S. Sun, "Wideband millimeter-wave propagation measurements and channel models for future wireless communication system design," IEEE Trans. Commun., vol. 63, no. 9, pp. 3029 - 3056, Sep. 2015.

[7] X. Zhao, S. Li, Q. Wang, M. Wang, S. Sun, and W. Hong, "Channel measurements, modeling, simulation and validation at $32 \mathrm{GHz}$ in outdoor microcells for $5 \mathrm{~g}$ radio systems," IEEE Access, vol. PP, no. 99, pp. $1-1,2017$.

[8] J. Ko, K. Lee, Y. J. Cho, S. Oh, S. Hur, N. G. Kang, J. Park, D. J. Park, and D. H. Cho, "Feasibility study and spatial-temporal characteristics analysis for $28 \mathrm{GHz}$ outdoor wireless channel modelling," IET Communications, vol. 10, no. 17, pp. $2352-2362,2016$.

[9] G. R. MacCartney, M. K. Samimi, and T. S. Rappaport, "Omnidirectional path loss models in new york city at $28 \mathrm{GHz}$ and $73 \mathrm{GHz}$," in IEEE 25th Annual International Symposium on Personal, Indoor, and Mobile Radio Communication (PIMRC), Sep. 2014, pp. 227 - 231.

[10] G. R. MacCartney, T. S. Rappaport, M. K. Samimi, and S. Sun, "Millimeter-wave omnidirectional path loss data for small cell 5G channel modeling," IEEE Access, vol. 3, pp. 1573 - 1580, 2015.

[11] A. Dammann, T. Jost, R. Raulefs, M. Walter, and S. Zhang, "Optimizing waveforms for positioning in 5G," in IEEE 17th International Workshop on Signal Processing Advances in Wireless Communications (SPAWC 2016), Edinburgh, United Kingdom, Jul. 2016.

[12] D. Dardari, A. Conti, U. Ferner, A. Giorgetti, and M. Win, "Ranging with ultrawide bandwidth signals in multipath environments," Proc. IEEE, vol. 97, no. 2, pp. $404-426$, Feb. 2009.

[13] C. Musso and J.-P. Ovarlez, "Improvement of the Ziv-Zakai lower bound for time delay estimation," in 15th European Signal Processing Conference (EUSIPCO 2007), Poznan, Poland, Sep. 2007, pp. $960-$ 964.

[14] T. Needham, "A visual explanation of jensen's inequality," The American Mathematical Monthly, vol. 100, no. 8, pp. 768 - 771, Oct. 1993.

[15] E. Staudinger and A. Dammann, "Sparse subcarrier allocation for timing-based ranging with OFDM modulated signals in outdoor environments," in Proceedings of $10^{\mathrm{th}}$ Workshop on Positioning Navigation and Communication, Dresden, Germany, Mar. 2013, pp. 2 - 2. 